\title{
Efficacy and safety of pemetrexed and nedaplatin followed by pemetrexed maintenance therapy in advanced lung adenocarcinoma
}

This article was published in the following Dove Press journal:

Cancer Management and Research

Number of times this article has been viewed

\author{
Zhong Lin ${ }^{1, *}$ \\ Wei-Ze Lvi,* \\ Si-Yang Wang ${ }^{2}$ \\ Jin-Lin Zou ${ }^{3}$ \\ Yun-Yan Con' \\ Zhi-Hui Wang' \\ Mei Xiao' \\ Pei-Jian Peng' \\ 'Department of Medical Oncology, \\ The Fifth Affiliated Hospital of \\ Sun-Yat-Sen University, Zhuhai, \\ Guangdong, People's Republic of \\ China; ${ }^{2}$ Department of Radiation \\ Oncology, The Fifth Affiliated Hospital \\ of Sun-Yat-Sen University, Zhuhai, \\ Guangdong, People's Republic of \\ China; ${ }^{3}$ Department of Surgical \\ Oncology, The Fifth Affiliated Hospital \\ of Sun-Yat-Sen University, Zhuhai, \\ Guangdong, People's Republic of \\ China \\ *These authors contributed equally to \\ this work
}

Correspondence: Pei-Jian Peng Department of Medical Oncology, The Fifth Affiliated Hospital of Sun-Yat-Sen University, 52 Mei Hua Road East, Zhuhai 519000, Guangdong, People's Republic of China

Email pengpjian@I63.com
Objective: To evaluate the efficacy and safety of pemetrexed and nedaplatin followed by pemetrexed maintenance therapy in advanced lung adenocarcinoma.

Methods: A total of 53 advanced lung adenocarcinoma patients hospitalized between July 2013 and June 2016 with a performance status $\leq 2$ were enrolled in this study. All patients received 4-6 cycles of combination chemotherapy comprising pemetrexed $\left(500 \mathrm{mg} / \mathrm{m}^{2} \mathrm{dL}\right)$ and nedaplatin $\left(80 \mathrm{mg} / \mathrm{m}^{2} \mathrm{dL}\right)$. Each chemotherapy cycle consisted of 21 days. After the efficacy of the combination chemotherapy was assessed, patients with stable disease, partial remission, or complete remission received pemetrexed maintenance therapy $\left(500 \mathrm{mg} / \mathrm{m}^{2} \mathrm{dL}\right)$ until disease progression or intolerable side effects occurred. Each pemetrexed maintenance therapy cycle was 28 days. Results: After completion of the pemetrexed and nedaplatin combination chemotherapy, 26 (49.1\%), 15 (28.3\%), and 12 (22.6\%) patients exhibited partial remission, stable disease, and progressive disease, respectively. Complete remission was not achieved in any patient. Therefore, the response and disease control percentages were $49.1 \%$ and $77.4 \%$, respectively. A total of 38 patients were further administered pemetrexed maintenance chemotherapy for an average of 9.8 cycles. The median progression-free survival and overall survival of the 38 patients receiving the pemetrexed maintenance therapy were 9.3 (95\% confidence interval: $8.6-10)$ months and 16.3 (95\% confidence interval: 14.5-18.2) months, respectively. The major adverse effects included bone marrow suppression and gastrointestinal reactions, which were well tolerated. Conclusions: Combination chemotherapy based on pemetrexed and nedaplatin is effective for the treatment of advanced lung adenocarcinoma with a high tolerance by patients. In addition, pemetrexed maintenance therapy of advanced lung adenocarcinoma is safe and effective for the treatment of advanced lung adenocarcinoma following pemetrexed and nedaplatin combination chemotherapy.

Keywords: lung adenocarcinoma, pemetrexed, nedaplatin, maintenance therapy

\section{Introduction}

Lung cancer is the most frequent cause of cancer mortality worldwide and the incidence of lung cancer in recent years has continued to rise. ${ }^{1}$ In China, lung cancer is the most common malignant tumor. ${ }^{2}$ A total of 733,300 new cases of lung cancer and 610,200 cases of lung cancer-related deaths were reported in China in 2015. Early diagnosis and treatment are effective approaches to improve the prognosis of lung cancer. However, lung cancer involves significant clinical heterogeneity, and the early detection and diagnosis of lung cancer are especially lacking in some developing countries. Most patients with lung cancer are diagnosed at advanced stages and cannot be treated surgically. ${ }^{3}$ Adenocarcinoma is the most common histopathological type 
of lung cancer. ${ }^{4}$ Epidermal growth factor receptor (EGFR) mutations have been targeted for effective treatment of lung adenocarcinoma. EGFR tyrosine kinase inhibitors have been surprisingly effective and have rapidly become the standard therapy for patients with EGFR mutations..$^{5}$ Also, tumors harboring anaplastic lymphoma kinase (ALK) and ROS1 fusions are highly sensitive to inhibitors targeting these kinases such as crizotinib. However, these drugs are not applicable to all adenocarcinoma patients because of the low incidence and detection rate of gene mutations or gene fusions..$^{5-7}$ Therefore, traditional chemotherapy agents are still the most common approach for treatment of lung adenocarcinoma, especially in China.

The National Comprehensive Cancer Network guidelines recommend platinum-based regimen chemotherapy as a firstline treatment for patients with advanced non-small cell lung cancer (NSCLC). However, disease progression has been reported in many patients within 3-6 months after stabilization with first-line chemotherapy. ${ }^{3,8}$ Maintenance treatment is therefore important prior to second-line therapy. Maintenance therapy refers to therapeutic approaches for cancer patients after first-line chemotherapy and before second-line chemotherapy, until the disease progresses or emergence of intolerable side effects. The purpose of maintenance therapy is to maximize remission and survival in cancer patients to further improve the efficacy and prognosis of cancer treatment. The drug selected for maintenance therapy must therefore have high efficacy, low toxicity, and convenience. The final outcomes of the PARAMOUNT trial showed that the median survival time was improved and the mortality rate was reduced, in non-squamous NSCLC patients who received pemetrexed maintenance therapy. ${ }^{8}$ Also, pemetrexed maintenance therapy improved progression-free survival (PFS) and overall survival (OS) following a non-pemetrexed-containing platinum doublet regimen. ${ }^{9}$

Previously, pemetrexed treatment was not covered by medical insurance in China and thus was not widely used in chemotherapy because of economic reasons. Since March 2013, pemetrexed has been covered by supplementary medical insurance in the "Supplementary Medical Insurance for Severe Diseases" guidelines announced by the Zhuhai Government, Guangdong Province, China. Pemetrexed has since been widely used in chemotherapy for lung adenocarcinoma in this region. This study was initially performed because of the wide application of pemetrexed treatment in clinics.

Nedaplatin, which was first approved in Japan in 1995, is a second-generation cytotoxic platinum drug, which shows no cross-resistance with traditional cisplatin and carboplatin. It has been reported that nedaplatin exhibits similar therapeutic effects but has 10 -fold greater water solubility and lower nephrotoxicity and gastrointestinal toxicity compared with cisplatin. The major dose-limiting toxicity of nedaplatin is bone marrow suppression, especially thrombocytopenia, leukopenia, and anemia. ${ }^{10}$ A number of clinical studies have reported significant therapeutic effects for nasopharyngeal carcinoma, esophageal cancer, and cervical cancer. ${ }^{11-13}$ During the past decade, nedaplatin chemotherapy has, therefore, gradually become the most commonly used chemotherapeutic agent for the treatment of NSCLC in China.

To determine the optimal chemotherapy regimen for the treatment of advanced lung adenocarcinoma, we designed the present study to evaluate the efficacy and safety of pemetrexed maintenance therapy for advanced lung adenocarcinoma following combination chemotherapy with pemetrexed and nedaplatin.

\section{Materials and methods Eligibility}

This study was reviewed and approved by the Ethics Committee of the Fifth Affiliated Hospital of Sun Yat-sen University. Written informed consent was obtained from all included patients. The trial was registered under the number ChiCTR-OIC-17011634. The inclusion criteria were 1) adult patients $>18$ years of age; 2 ) newly diagnosed cases without chemotherapy; 3) stage IIIb-IV lung adenocarcinoma confirmed by histopathological examinations and measurable tumor lesions; 4) a performance status (PS) of 0-2 based on the Eastern Cooperative Oncology Group scoring system; 5) >3 months expected survival; 6) normal physiological functions including an absolute neutrophil count $\geq 1.5 \times 10^{9} / \mathrm{L}$, hemoglobin level $\geq 80 \mathrm{~g} / \mathrm{L}$, platelet count $\geq 75 \times 10^{9} / \mathrm{L}$, total bilirubin level $\leq 1.5$-fold the upper limit of normal (ULN), aspartate aminotransferase and alanine aminotransferase levels $\leq 2.5$-fold the ULN, serum creatinine level $\leq 1.5$-fold the ULN; and 7) no brain metastases.

The exclusion criteria were 1) breastfeeding and/or pregnant patients; 2) systematic diseases including acute infections, major cardiovascular disease such as myocardial infarction over the past 1 year, serious liver diseases, serious kidney diseases, and metabolic disorders; 3 ) other malignant tumors during the last 5 years, except for malignant tumors that can be treated by radical resection, including in situ cervical cancer, basal or squamous cell skin cancer, breast in situ intraductal carcinoma and localized prostate cancer; and 4) viral diseases that may be transmitted via the blood or 
other body fluids, such as human immunodeficiency virus, hepatitis B, or hepatitis C.

\section{Chemotherapy}

A combination chemotherapy cycle included 21 days of intravenous infusion of pemetrexed $\left(500 \mathrm{mg} / \mathrm{m}^{2} \mathrm{dL}\right)$ and nedaplatin $\left(80 \mathrm{mg} / \mathrm{m}^{2} \mathrm{dL}\right)$. Each patient underwent $4-6$ cycles of the combination chemotherapy. The chemotherapy was terminated if intolerable adverse effects occurred. The efficacy and safety were assessed after 4-6 cycles of chemotherapy. Patients with stable disease (SD), partial remission (PR), or complete remission $(\mathrm{CR})$ received pemetrexed maintenance therapy $\left(500 \mathrm{mg} / \mathrm{m}^{2} \mathrm{dL}\right.$ pemetrexed; 28 days/cycle) until disease progression or intolerable side effects occurred. The efficacy and safety were assessed according to the response rate (RR), disease control rate (DCR), PFS, OS, and adverse events. Folic acid and vitamin B12 were supplemented during the chemotherapy. Dexamethasone was used to prevent pemetrexed-induced rash. Conventional antiemetic treatment was administered before and after the chemotherapy.

\section{Assessments of efficacy and safety}

The primary outcomes of this clinical trial were the RR, PFS, and toxicities. Imaging reviews and tumor lesion measurements were conducted every 2 cycles. Blood tests, liver and kidney function tests, and electrocardiography were performed to assess the adverse effects of the chemotherapeutic agents. According to the Response Evaluation Criteria in Solid Tumors, version 1.1, chemotherapy efficacy is classified as either a CR, PR, SD, or progressive disease. Toxicities were assessed using the National Cancer Institute Common Toxicity Criteria (version 3.0) before each treatment cycle. If the toxicity caused a chemotherapy delay of more than 3 weeks, the patient withdrew from the trial.

\section{Dosage adjustments}

The chemotherapy dosage was adjusted if severe adverse effects (>grade III toxicity) were reported during chemotherapy. The chemotherapy dosage was reduced $20 \%$ or $40 \%$ if grade III or IV toxicity occurred, respectively. The chemotherapy regimen was terminated if the dosage had to be reduced by $>40 \%$.

\section{Statistical analyses}

OS, overall response rate, and PFS were assessed. All patients had toxicity assessments in at least 2 cycles of chemotherapy. Statistical analyses were conducted using SPSS statistical software for Windows, version 17.0 (SPSS, Chicago, IL,
USA). The Kaplan-Meier method was used to analyze the survival data.

\section{Results}

\section{Patient characteristics}

A total of 53 advanced lung adenocarcinoma patients (38 males and 15 females) hospitalized between July 2013 and June 2016 were enrolled in this prospective study. The patient characteristics are shown in Table 1 . The age of the 53 patients ranged between 33 and 73 (median age: 60) years. Of the 53 patients, 45 had a smoking history and 8 had no smoking history. Forty-seven patients had a PS score of $0-1$ and 6 patients had a PS score of 2. Based on histopathological examinations, 5 and 48 patients were classified as stage IIIlb and stage IV, respectively.

\section{Chemotherapy efficacy}

All patients completed over 2 cycles of first-line chemotherapy with assessable chemotherapeutic efficacy. A CR was not achieved in any patient, while $26(49.1 \%), 15$ (28.3\%), and $12(22.6 \%)$ patients exhibited PR, SD, and progressive disease, respectively. The RR and DCR were $49.1 \%$ and $77.4 \%$, respectively (Table 2). Forty-one patients were eligible for pemetrexed maintenance therapy; however, 3 of these patients refused maintenance therapy because of economic reasons. Thus, a total of 38 patients received pemetrexed maintenance therapy for an average of 9.8 (range: 4-52) cycles. The median PFS and OS of the 38 patients who underwent pemetrexed maintenance therapy were 9.3 (95\% confidence interval

Table I Patient characteristics

\begin{tabular}{lll}
\hline Characteristics & Patients (n) & Patients (\%) \\
\hline Total & $\mathbf{5 3}$ & 100 \\
Sex & & \\
Male & 38 & 71.7 \\
Female & 15 & 28.3 \\
Age (years) & & \\
Median & 60 & \\
Range & $33-73$ & \\
ECOG performance status & & 15.1 \\
0 & 8 & 73.6 \\
I & 39 & 11.3 \\
2 & 6 & \\
Staging & & 9.4 \\
Illb & 5 & 90.6 \\
IV & 48 & 84.9 \\
Smoking exposure & & 15.1 \\
Smoking & 45 & \\
No smoking & 8 & \\
\hline
\end{tabular}

Abbreviation: ECOG, Eastern Cooperative Oncology Group. 
[CI]: 8.6-10) months and 16.3 (95\% CI: 14.5-18.2) months, respectively (Figures 1 and 2). The median PFS and OS of all 53 patients were 8.9 (95\% CI: 8.3-9.5) months and 14.5 (95\% CI: 13.2-16.2) months, respectively (Figures 3 and 4).

\section{Adverse effects of chemotherapy}

Most adverse effects were reversible. The most common adverse effect was bone marrow suppression. During the combination chemotherapy, grade III leukocyte suppression was observed in 6 patients (11.3\%), but no grade IV leukocyte suppression was reported. A total of $5(9.4 \%)$ patients had grade III anemia. No grade IV anemia or greater than grade II platelet inhibition was reported. No grade III/IV non-bone marrow toxicity was found. Grade I allergic reactions induced by nedaplatin were reported in 2 patients. Other adverse effects were mild and tolerable, and no treatment-related death was reported (Table 2).

Table 2 The side effects of chemotherapy regimen of the combination of pemetrexed and nedaplatin for the treatment of advanced lung adenocarcinoma

\begin{tabular}{lllll}
\hline Side effects & \multicolumn{4}{l}{ Toxicity grade (WHO criteria) } \\
\cline { 2 - 5 } & I & II & III & IV \\
\hline Neutropenia & $15(28.3 \%)$ & $6(11.3 \%)$ & $6(11.3 \%)$ & 0 \\
Thrombocytopenia & $9(17 \%)$ & $5(9.4 \%)$ & 0 & 0 \\
Hemoglobin reduction & II (20.8\%) & $6(11.3 \%)$ & $5(9.4 \%)$ & 0 \\
Nausea/vomiting & $14(26.4 \%)$ & $9(17 \%)$ & 0 & 0 \\
Allergic reactions & $2(3.8 \%)$ & 0 & 0 & 0 \\
Liver damage & $6(11.3 \%)$ & 0 & 0 & 0 \\
Renal impairment & $2(3.8 \%)$ & 0 & 0 & 0 \\
Hair loss & $12(22.6 \%)$ & $4(7.5 \%)$ & 0 & 0 \\
\hline
\end{tabular}

Abbreviation: WHO, World Health Organization.

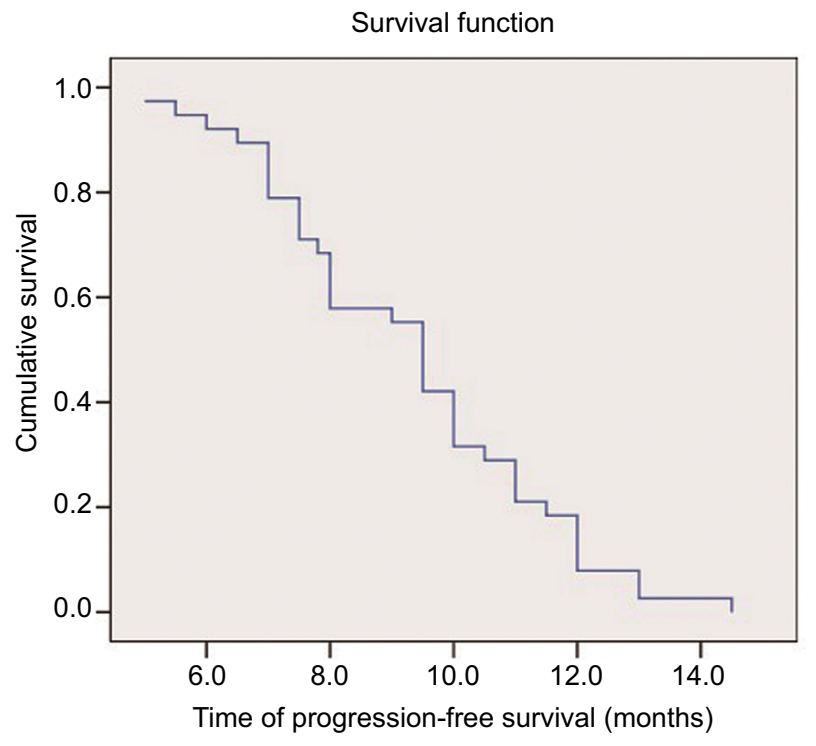

Figure I Kaplan-Meier curve of progression-free survival of the 38 patients who underwent pemetrexed maintenance therapy.

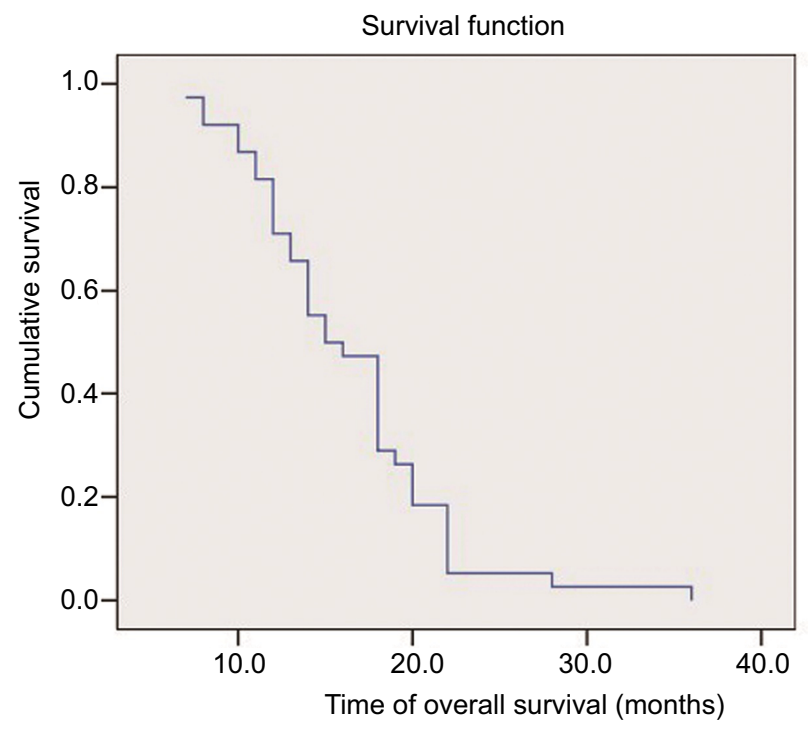

Figure 2 Kaplan-Meier curve of overall survival of the 38 patients who underwent pemetrexed maintenance therapy.

During pemetrexed maintenance therapy, 4 patients (10.5\%) exhibited grade III leukocyte suppression and no patient had grade IV leukocyte suppression. In addition, 2 patients had grade III anemia. No grade IV anemia or greater than grade II platelet inhibition was found. No grade III/IV non-bone marrow toxicity was identified. Other adverse effects were mild and tolerable, and no treatment-related death was reported (Table 3). Eight (21\%) patients underwent pemetrexed dosage adjustment (decreased dosage or delayed treatment). No patient received a red blood cell transfusion, $10.5 \%$ received erythropoietin, $15 \%$ received granulocyte colony stimulating factor, and none received a platelet transfusion (Table 3).

\section{Discussion}

In the present study, we assessed the efficacy and safety of pemetrexed and nedaplatin combination chemotherapy in 53 cases of advanced lung adenocarcinoma. This chemotherapeutic regimen resulted in an RR of $49.1 \%$ and a DCR of $77.4 \%$. In addition, all patients tolerated the chemotherapy side effects, which was especially important in those patients completing the chemotherapy program and undergoing further maintenance chemotherapy. After first-line pemetrexed and nedaplatin chemotherapy, we administered pemetrexed maintenance chemotherapy for 28 days to evaluate the efficacy and safety. A total of 38 advanced lung adenocarcinoma cases were included in the present study, and each patient received an average of 9.8 cycles of pemetrexed-based maintenance therapy. The median PFS and OS of the patients who received pemetrexed maintenance therapy were 9.3 (95\% 


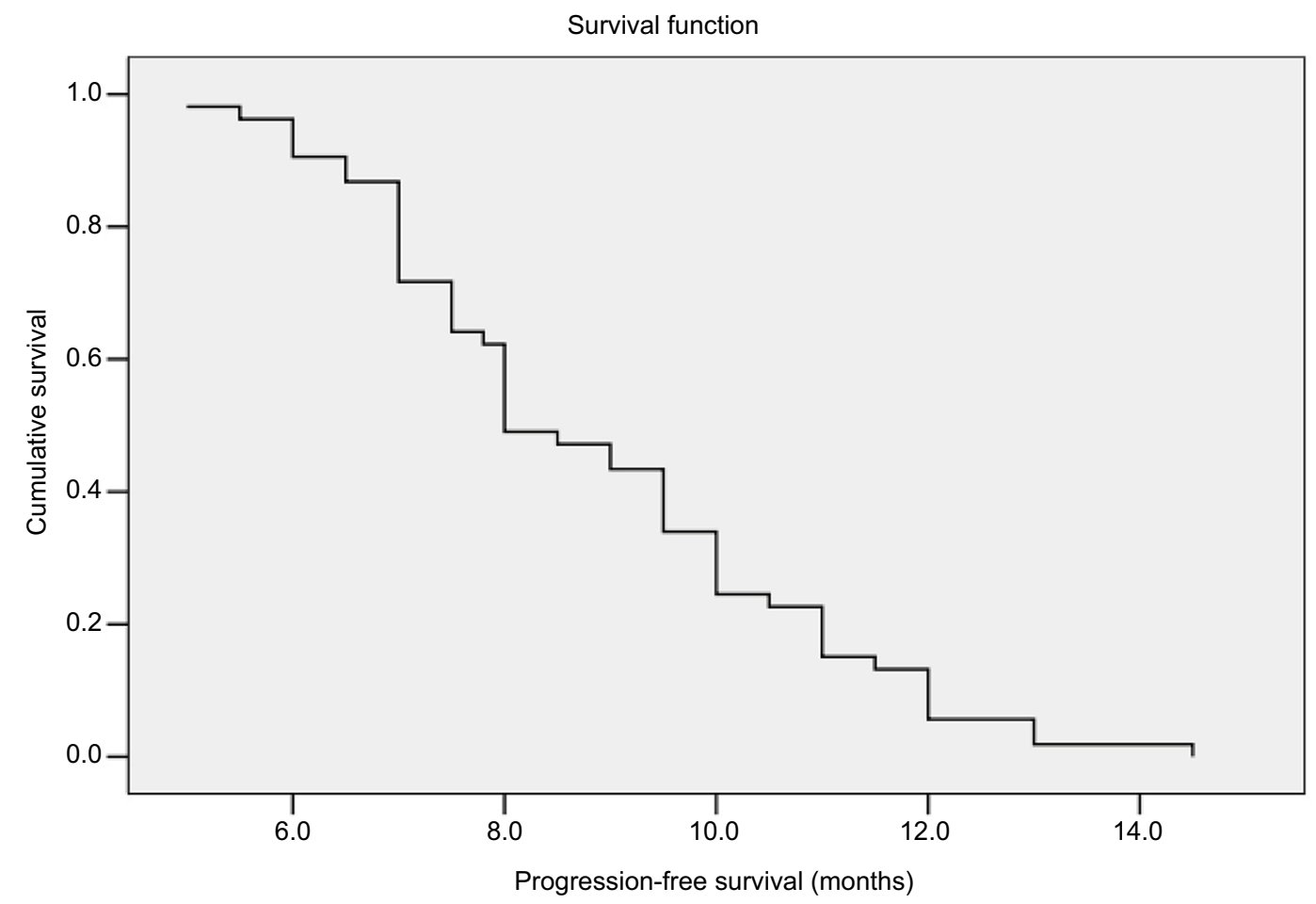

Figure 3 Kaplan-Meier curve of progression-free survival of all 53 patients.

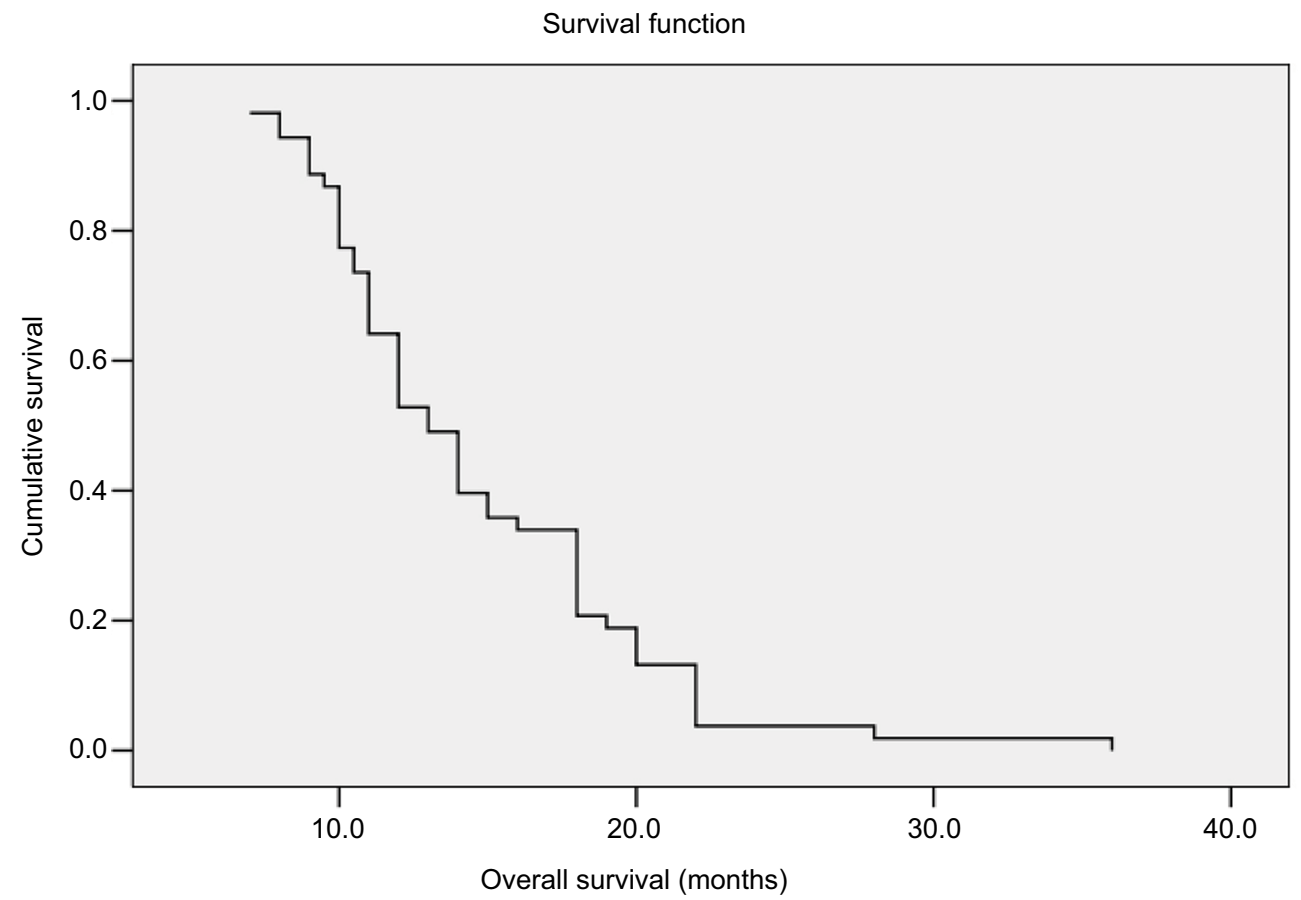

Figure 4 Kaplan-Meier curve of overall survival of all 53 patients.

CI: 8.6-10) months and 16.3 (95\% CI: 14.5-18.2) months, respectively. All patients tolerated the adverse effects, including hematological toxicity, gastrointestinal reactions, liver and kidney dysfunctions, muscle soreness, and hair loss, which were minimized by symptomatic treatment and did not affect the chemotherapy regimen.

Combination chemotherapy based on cisplatin is the most effective chemotherapy regimen for the treatment of NSCLC. ${ }^{14}$ 
Table 3 The side effects of pemetrexed maintenance therapy of advanced lung adenocarcinoma following combination chemotherapy of nedaplatin and pemetrexed

\begin{tabular}{lllll}
\hline Side effects & \multicolumn{4}{l}{ Toxicity grade (WHO criteria) } \\
\cline { 2 - 5 } & I & II & III & IV \\
\hline Neutropenia & $12(31.6 \%)$ & $4(10.5 \%)$ & $4(10.5 \%)$ & 0 \\
Thrombocytopenia & $5(13.2 \%)$ & $4(10.5 \%)$ & 0 & 0 \\
Hemoglobin reduction & $10(26.3 \%)$ & $6(15.8 \%)$ & $2(5.5 \%)$ & 0 \\
Nausea/vomiting & II (28.9\%) & $5(13.2 \%)$ & 0 & 0 \\
Allergic reactions & 0 & 0 & 0 & 0 \\
Liver damage & $4(10.5 \%)$ & 0 & 0 & 0 \\
Renal impairment & 0 & 0 & 0 & 0 \\
Hair loss & $9(23.7 \%)$ & $3(7.9 \%)$ & 0 & 0 \\
\hline
\end{tabular}

Abbreviation: WHO, World Health Organization.

However, cisplatin may cause a number of serious adverse effects, such as gastrointestinal reactions, nephrotoxicity, neurotoxicity, and myelosuppression. As the most significant dose-limiting response, ${ }^{15}$ nephrotoxicity can be minimized by extensive hydration of cisplatin and diuretics following cisplatin use. ${ }^{16,17}$ Numerous cancer patients are forced to discontinue cisplatin-based chemotherapy regimens because of severe adverse effects caused by cisplatin. Therefore, a number of cisplatin derivatives have been developed and used in chemotherapy. Nedaplatin is a second-generation platinumbased antitumor agent, ${ }^{18}$ which has a higher water solubility, lower toxicity, but similar therapeutic effects compared with cisplatin. Koshiyama et al reported that nedaplatin exhibited similar or better tumor inhibition than did cisplatin (cervical cancer: $70.7 \%$ versus $63.9 \%, n=15$; ovarian cancer: $61.7 \%$ versus $54.8 \%, \mathrm{n}=65$; and endometrial carcinoma: $52.1 \%$ versus $47.7 \%, n=57) .{ }^{13}$ As a multi-target folate antagonist, ${ }^{19}$ pemetrexed blocks cell division at the $\mathrm{S}$ phase by inhibiting dihydrofolate reductase, thymidylate synthase, and glycinamide ribonucleotide formyltransferase, which are involved in purine synthesis. In 2004, the US Food and Drug Administration (FDA) first approved the combination of pemetrexed and cisplatin as first-line treatment for malignant pleural mesothelioma and second-line treatment for NSCLC. ${ }^{20,21}$ Furthermore, it has been reported that pemetrexed is effective as either first-line, second-line, or maintenance therapy for NSCLC. ${ }^{8,21,22}$

Since 2009, the US FDA has approved pemetrexed as a maintenance therapy for advanced non-squamous NSCLC.22 The efficacy of pemetrexed depends on the histopathological type of NSCLC. Pemetrexed exhibited significant efficacy for the treatment of non-squamous NSCLC but not squamous NSCLC. ${ }^{8}$ Non-squamous NSCLC cases were therefore enrolled in the present study to assess the efficacy and safety of pemetrexed and nedaplatin. We assessed the RR and DCR of 53 NSCLC patients after completion of the chemotherapy regimen and found an RR of $49.1 \%$ and a DCR of $77.4 \%$, with no severe adverse effects observed. The adverse effects were relieved by symptomatic treatments, and the chemotherapy regimen was not affected by any adverse effects. Taken together, our results are consistent with most previous studies that used a combination of pemetrexed and cisplatin for the treatment of advanced NSCLC. ${ }^{23-25}$

The PARAMOUNT trial results reported a median survival of 13.9 months in non-squamous NSCLC patients receiving pemetrexed maintenance therapy, which was significantly higher than that (11.0 months) in the placebo group (hazard ratio $=0.78, P=0.0195$ ), suggesting that pemetrexed maintenance therapy improved the median survival by 2.9 months. ${ }^{8} \mathrm{In}$ addition, the risk of death was reduced by $22 \%$ by pemetrexed maintenance therapy (95\% CI: 0.64-0.96). Our results showed that the mean PFS and median survival of 38 non-squamous NSCLC patients were 9.3 (95\% CI: 8.6-10) months and 16.3 (95\% CI: 14.5-18.2) months, respectively, which were similar to the PARAMOUNT trial results. In the PARAMOUNT trial, pemetrexed was used once for 21 days $\left(500 \mathrm{mg} / \mathrm{m}^{2}\right)$, and patients received an average of 7.9 cycles of pemetrexed maintenance chemotherapy, which was terminated due to disease progression and/or intolerable side effects. In the present study, the 38 patients received an average of 5.8 cycles of pemetrexed maintenance chemotherapy, and pemetrexed was used once for 28 days. Therefore, the interval of the chemotherapy cycle in the present study was longer than that in the PARAMOUNT trial study. Typically, fewer adverse effects caused by chemotherapy agents contribute to better efficacy, because patients can tolerate a longer chemotherapy term.

After first-line chemotherapy, 38 patients were further treated with pemetrexed maintenance chemotherapy, excluding 3 patients who refused maintenance chemotherapy because of economic reasons. The percentage of patients receiving pemetrexed maintenance chemotherapy in the present study was significantly higher than that in other regions in China, which suggests that the medical insurance policy has significantly influenced the clinical outcomes.

While our results showed a limited improvement in the clinical outcomes of lung adenocarcinoma patients, the chemotherapy regimen exhibited high safety and clinical tolerance. Our study, therefore, strongly suggested that first-line combination chemotherapy consisting of pemetrexed and nedaplatin followed by pemetrexed maintenance chemotherapy can further improve the quality of life of lung adenocarcinoma patients. However, the limitations of the present study included a small sample size and no control group, and thus randomized and carefully controlled studies involving more patients are needed. 


\section{Acknowledgment}

We thank all of the patients and their families for their willingness to take part in this study.

\section{Disclosure}

The authors report no conflicts of interest in this work.

\section{References}

1. Torre LA, Bray F, Siegel RL, et al. Global cancer statistics, 2012. CA Cancer J Clin. 2015;65(2):87-108.

2. Chen W, Zheng R, Baade PD, et al. Cancer statistics in China, 2015. CA Cancer J Clin. 2016;66(2):115-132.

3. NSCLC Meta-Analyses Collaborative Group. Chemotherapy in addition to supportive care improves survival in advanced non-small-cell lung cancer: a systematic review and meta-analysis of individual patient data from 16 randomized controlled trials. J Clin Oncol. 2008;26(28):4617-4625.

4. Cancer Genome Atlas Research Network. Comprehensive molecular profiling of lung adenocarcinoma. Nature. 2014;511(7511): 543-550.

5. Ding L, Getz G, Wheeler DA, et al. Somatic mutations affect key pathways in lung adenocarcinoma. Nature. 2008;455(7216):1069-1075.

6. Kwak EL, Bang YJ, Camidge DR, et al. Anaplastic lymphoma kinase inhibition in non-small-cell lung cancer. $N$ Engl J Med. 2010;363(18):1693-1703.

7. Chin LP, Soo RA, Soong R, Ou SH. Targeting ROS1 with anaplastic lymphoma kinase inhibitors: a promising therapeutic strategy for a newly defined molecular subset of non-small-cell lung cancer. JThorac Oncol. 2012; 7(11):1625-1630.

8. Pazares LG, De MF, Dediu M, et al. PARAMOUNT: Final overall survival results of the phase III study of maintenance pemetrexed versus placebo immediately after induction treatment with pemetrexed plus cisplatin for advanced nonsquamous non-small-cell lung cancer. J Clin Oncol. 2013;31(23):2895-2902.

9. Ciuleanu T, Brodowicz T, Zielinski C, et al. Maintenance pemetrexed plus best supportive care versus placebo plus best supportive care for non-small-cell lung cancer: A randomised, double-blind, phase 3 study. Lancet. 2009; 374(9699):1432-1440.

10. Tsuda H, Hashiguchi Y, Nishimura S, et al. Phase I-II study of irinotecan (CPT-11) plus nedaplatin (254-S) with recombinant human granulocyte colony-stimulating factor support in patients with advanced or recurrent cervical cancer. Br J Cancer. 2004;91(6):1032-1037.
11. Kodama J, Takemoto M, Seki N, et al. Phase I study of weekly nedaplatin and concurrent pelvic radiotherapy as adjuvant therapy after radical surgery for cervical cancer. Int J Gynecol Cancer. 2008;18(5):1037-1041.

12. Cao W, Xu C, Lou G, et al. A phase II study of paclitaxel and nedaplatin as first-line chemotherapy in patients with advanced esophageal cancer. Jpn J Clin Oncol. 2009;39(9):582-587.

13. Koshiyama M, Kinezaki M, Uchida T, Sumitomo M. Chemosensitivity testing of a novel platinum analog, nedaplatin (254-S), in human gynecological carcinomas: a comparison with cisplatin. Anticancer Res. 2005;25(6C):4499-4502.

14. Rinaldi M, Cauchi C, Gridelli C. First line chemotherapy in advanced or metastatic NSCLC. Ann Oncol. 2006;17(Suppl 5):v64-v67.

15. Boulikas T, Vougiouka M. Cisplatin and platinum drugs at the molecular level. Oncol Rep. 2003;10(6):1663-1682.

16. Ozkok A, Edelstein CL. Pathophysiology of cisplatin-induced acute kidney injury. Biomed Res Int. 2014;2014:967826.

17. Arany I, Safirstein RL. Cisplatin nephrotoxicity. Semin Nephrol. 2003;23(5):460-464.

18. Kurata T, Tamura K, Yamamoto N, et al. Combination phase I study of nedaplatin and gemcitabine for advanced non-small-cell lung cancer. Br J Cancer. 2004;90(11):2092-2096.

19. Baldwin CM, Perry CM. Pemetrexed: a review of its use in the management of advanced non-squamous non-small cell lung cancer. Drugs. 2009;69(16):2279-2302.

20. Hazarika M, White RM, Johnson JR, Pazdur R. FDA drug approval summaries: pemetrexed (Alimta). Oncologist. 2004;9(5):482-488.

21. Cohen MH, Johnson JR, Wang YC, Sridhara R, Pazdur R. FDA drug approval summary: pemetrexed for injection (Alimta) for the treatment of non-small cell lung cancer. Oncologist. 2005;10(6):363-368.

22. Cohen MH, Cortazar P, Justice R, Pazdur R. Approval summary: pemetrexed maintenance therapy of advanced/metastatic nonsquamous, nonsmall cell lung cancer (NSCLC). Oncologist. 2010;15(12):1352-1358.

23. Mubarak N, Gaafar R, Shehata S, et al. A randomized, phase 2 study comparing pemetrexed plus best supportive care versus best supportive care as maintenance therapy after first-line treatment with pemetrexed and cisplatin for advanced, non-squamous, non-small cell lung cancer. BMC Cancer. 2012;12:423.

24. Scagliotti GV, Parikh P, von Pawel J, et al. Phase III study comparing cisplatin plus gemcitabine with cisplatin plus pemetrexed in chemotherapy-naive patients with advanced-stage non-small cell lung cancer. J Clin Oncol. 2008;26(21):3543-3551.

25. Zhang X, Lu J, Xu J, et al. Pemetrexed plus platinum or gemcitabine plus platinum for advanced non-small cell lung cancer: final survival analysis from a multicentre randomized phase II trial in the East Asia region and a meta-analysis. Respirology. 2013;18(1):131-139.
Cancer Management and Research

\section{Publish your work in this journal}

Cancer Management and Research is an international, peer-reviewed open access journal focusing on cancer research and the optimal use of preventative and integrated treatment interventions to achieve improved outcomes, enhanced survival and quality of life for the cancer patient. The manuscript management system is completely online and includes

\section{Dovepress}

a very quick and fair peer-review system, which is all easy to use. Visit http://www.dovepress.com/testimonials.php to read real quotes from published authors. 